\title{
Machine or Human? Evaluating the Quality of a Language Translation Mobile App for Diabetes Education Material
}

\author{
Xuewei Chen ${ }^{1}$, CHES, MEd, PhD; Sandra Acosta ${ }^{2}$, PhD; Adam E Barry ${ }^{3}$, PhD \\ ${ }^{1}$ Transdisciplinary Center for Health Equity Research, Department of Health and Kinesiology, Texas A\&M University, College Station, TX, United \\ States \\ ${ }^{2}$ Texas A\&M University, Department of Educational Psychology, Texas A\&M University, College Station, TX, United States \\ ${ }^{3}$ Texas A\&M University, Department of Health and Kinesiology, Texas A\&M University, College Station, TX, United States
}

\section{Corresponding Author:}

Xuewei Chen, CHES, MEd, PhD

Transdisciplinary Center for Health Equity Research

Department of Health and Kinesiology

Texas A\&M University

Blocker 311B

4243 TAMU

College Station, TX, 77843

United States

Phone: 19796760758

Fax: 19798478987

Email: xueweichen@tamu.edu

\section{Abstract}

Background: Diabetes is a major health crisis for Hispanics and Asian Americans. Moreover, Spanish and Chinese speakers are more likely to have limited English proficiency in the United States. One potential tool for facilitating language communication between diabetes patients and health care providers is technology, specifically mobile phones.

Objective: Previous studies have assessed machine translation quality using only writing inputs. To bridge such a research gap, we conducted a pilot study to evaluate the quality of a mobile language translation app (iTranslate) with a voice recognition feature for translating diabetes patient education material.

Methods: The pamphlet, "You are the heart of your family...take care of it," is a health education sheet for diabetes patients that outlines three recommended questions for patients to ask their clinicians. Two professional translators translated the original English sentences into Spanish and Chinese. We recruited six certified medical translators (three Spanish and three Chinese) to conduct blinded evaluations of the following versions: (1) sentences interpreted by iTranslate, and (2) sentences interpreted by the professional human translators. Evaluators rated the sentences (ranging from 1-5) on four scales: Fluency, Adequacy, Meaning, and Severity. We performed descriptive analyses to examine the differences between these two versions.

Results: Cronbach alpha values exhibited high degrees of agreement on the rating outcomes of both evaluator groups: 920 for the Spanish raters and .971 for the Chinese raters. The readability scores generated using MS Word's Flesch-Kincaid Grade Level for these sentences were 0.0, 1.0, and 7.1. We found iTranslate generally provided translation accuracy comparable to human translators on simple sentences. However, iTranslate made more errors when translating difficult sentences.

Conclusions: Although the evidence from our study supports iTranslate's potential for supplementing professional human translators, further evidence is needed. For this reason, mobile language translation apps should be used with caution.

(JMIR Diabetes 2017;2(1):e13) doi: 10.2196/diabetes.7446

\section{KEYWORDS}

health literacy; health education; health communication; language translation; diabetes; machine translation; mobile translation app; human interpreter; translator 


\section{Introduction}

Diabetes is a major health crisis for Hispanics and Asian Americans. According to the Centers for Disease Control and Prevention (CDC), 29.1 million people (9.3\% of the US population) have diabetes; $12.8 \%$ Hispanics and 9\% Asian Americans above 20 years old were diagnosed with diabetes, compared to 7.6\% non-Hispanic whites [1]. From 1997-2014, diabetes rates increased 103\% for Asian Americans and 60\% for Hispanics [2].

Compared to other ethnic groups, Hispanics and Chinese Americans are also more likely to have low English proficiency. Over $21 \%$ of the US population speaks a language other than English at home. Further, the highest percentages of individuals who speak no English are Hispanics and Chinese Americans [3]. Approximately $43.7 \%$ Hispanics and $55.7 \%$ Chinese Americans speak English less than "very well" [3] and would be considered having limited English proficiency (LEP). LEP refers to any person age 5 or older who self-reported speaking English less than "very well" [3]. In brief, because Hispanic and Chinese Americans are more likely to have LEP, communication challenges arising from language barriers might impact the quality of the health services and information they receive.

Populations with LEP encounter numerous health communication challenges due to barriers related to language proficiency. These language barriers, as many studies have pointed out, might lead to health disparities and poor health outcomes. For instance, individuals with LEP are more likely to take inaccurate medication dosages [4], have poor health status [5], spend additional money and time utilizing health care services [6], experience unsatisfactory events with health care providers, make improper health choices [7], and have limited access and use of preventive health care services [8]. For diabetes patients who have LEP, negative health outcomes include poor glycemic control [9] and diabetic retinopathy [10].

One potential tool for facilitating language communication between patients and health care providers is technology, specifically mobile phones. In the United States, smartphone ownership increased from $35 \%$ of the population in 2011 to $72 \%$ in 2016 [11]. These smartphone owners can access various apps including machine language translation apps. For instance, iTranslate is a mobile app available for mobile phones with Apple, Android, and Windows systems that instantly translates text or voice inputs and converts them into text and voice outputs. Such voice recognition features were developed from computerized systems.

There are no significant differences in smartphone ownership among different racial/ethnic groups [12]. Further, about three-quarters $(73 \%)$ of the Hispanic smartphone owners have used their phones to search for health-related information, compared to $58 \%$ white and $67 \%$ black [13]. Smartphones with machine translation apps are efficient tools for helping populations with LEP overcome language barriers [14,15]. For instance, translation mobile apps might improve their understanding of health information and access to health resources.
However, translation inaccuracy has the potential to adversely impact information's meaning and lead to negative health consequences. For example, language translation errors lead to misunderstandings about medical prescriptions [16] as well as misdiagnoses and mistreatments $[17,18]$.

Previous studies have examined the usability of mobile language translation apps among clinicians and patients. In a study conducted by Abreu and Adriatico [19], the researchers investigated the experience of using the Google Translation App among a group of US audiologists and Spanish speaking patients/parents/guardians when they were communicating with each other. Abreu and Adriatico reported positive reactions from both the audiologists and the Spanish-speaking clients. Based on their findings, the authors concluded that the Google Translation App might be a viable tool for addressing language barriers and improving health communication when human interpreters were not available [19]. Similarly, Albrecht et al [20] examined the usage experience of a mobile translation app (xprompt) among nursing staff in Germany. The authors found that the participants perceived the xprompt app as useful for basic communication with non-German speaking patients [20]. Here, machine translation refers to automated computer translations powered by algorithms.

Besides usability, accuracy is another important criterion for evaluating machine language translation tools. With regard to the machine translation accuracy, previous studies assessed the translation product provided by Babel Fish and Google Translate websites using only writing inputs [21-24]. They noted that machine translation tools made errors when translating medical information [21-24]. Khanna et al [22] suggested that machine translation tools with a voice recognition feature might increase translation errors. Given the absence of research on voice recognition features and translations errors, we investigated the quality of a machine language translation mobile app with a voice recognition feature (iTranslate). Because diabetes is a major health crisis for Hispanics and Asian Americans [2], we selected diabetes patient education material. To the best of our knowledge, no study to date has investigated the quality of a mobile translation app interpreting spoken sentences.

The purpose of this pilot study is to evaluate the quality of iTranslate when interpreting spoken sentences from English to Spanish and Chinese. Our overarching research question is: Can iTranslate be an accurate and practical translation tool for patients-clinicians using diabetes education materials? To provide insights into this question, we posed the following research questions:

1. What is the quality of iTranslate when interpreting spoken sentences from English to Spanish, as compared to professional human interpreters?

2. What is the quality of iTranslate when interpreting spoken sentences from English to Chinese, as compared to professional human translators? 


\section{Methods}

\section{Materials to be Translated}

We chose a publicly available diabetes patient education pamphlet as a heuristic example for this pilot study. The pamphlet, "You are the heart of your family...take care of it" (see Multimedia Appendix 1), is published by the National Institutes of Health and the CDC and distributed by the National Diabetes Education Program. This pamphlet contains two parts: Part A, six written sentences as behavior change suggestions for managing diabetes and Part B, three recommended questions for patients to ask their clinicians. Our study examined the quality of iTranslate when translating Part B. The study and results of Part A were reported by Chen et al [21].

\section{Procedures}

This study was approved by the appropriate institutional review board. Figure 1 outlines the procedures employed throughout this study, which comprise four steps: Step 1. iTranslate mobile app translation process; Step 2. Human translation process; Step 3. Voice transformation; Step 4. Evaluation.

Figure 1. Four-step procedures.

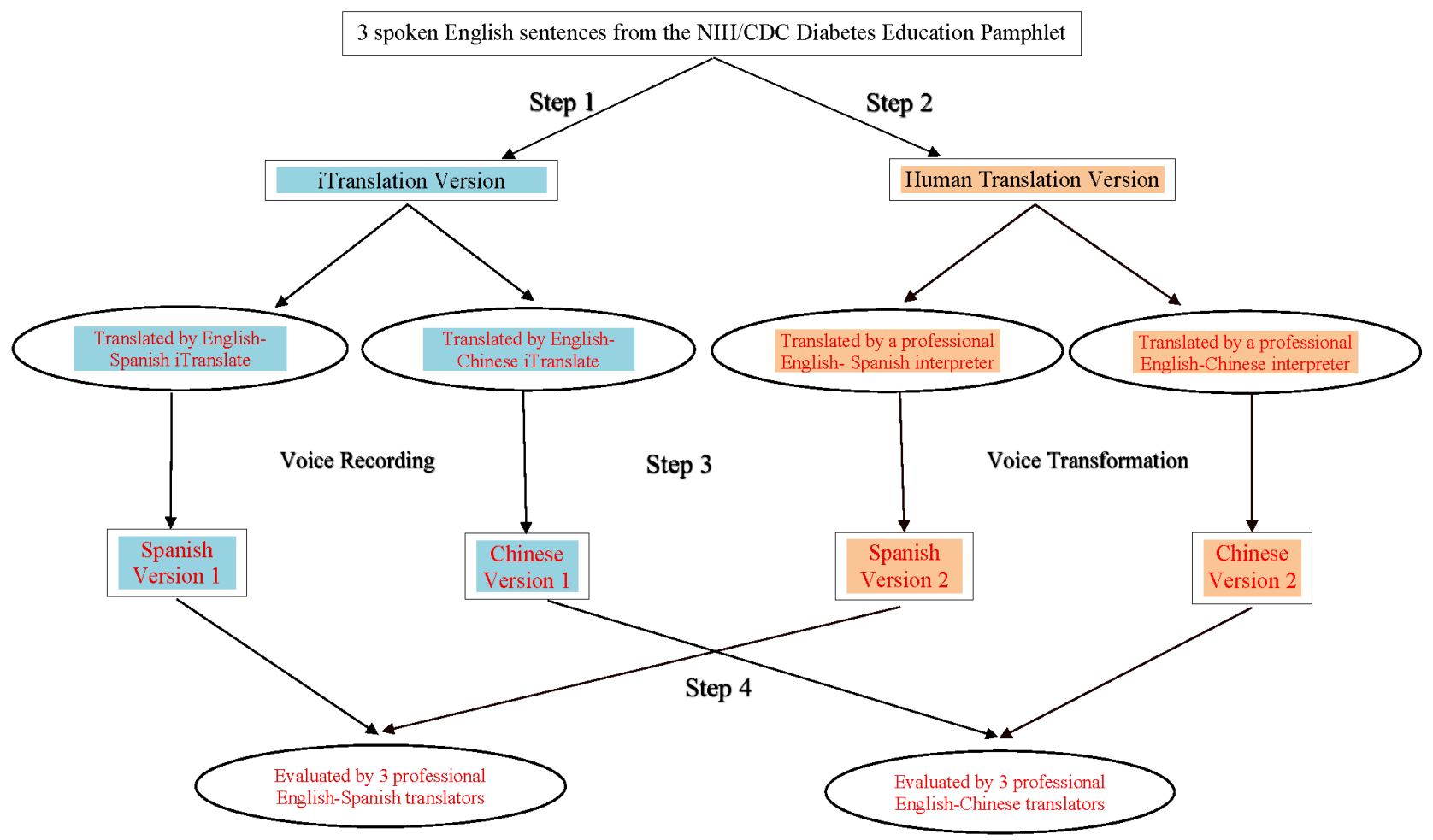

\section{Step 1. Mobile Language Translation App}

We used iTranslate app to translate three spoken questions from English into both Spanish and Chinese (Mandarin). We recorded these voice outputs into audio files.

\section{Step 2. Human Translator}

Two professional medical interpreters translated the three original English questions into Spanish and Chinese respectively. Both were American Translators Association (ATA) certified translators (one certified in English to Spanish and the other in English to Chinese). The ATA website lists all the certified translators' contact information. We approached the translators as regular customers seeking and paying for translation services. We did not inform them that their translations would be evaluated. We emailed the original English questions to the translators, and they returned the translated sentences in audio files by email as well. We also asked them to provide transcriptions of their voice translations in a separate MS Word file.

\section{Step 3. Voice Transformation}

Since the evaluators might distinguish the machine from the human translation because of the potentially recognizable characteristics of the mechanical voice, we converted all the human voice translations into a machine voice. First, we copied and pasted the transcription on a voice transformation website and clicked the voice button for the three questions translated by the human interpreters to be converted to the machine voice function. Second, we reviewed the transcriptions and compared the machine voice to the original human voice (translators' audio files) to ensure equivalency. Third, we recorded the three sentences, now in the machine voice, into audio files, and emailed these audio files to the evaluators. Table 1 lists the original English sentences and the translated Spanish and Chinese transcriptions. 
Table 1. The original English and translated Chinese and Spanish versions of the sentences.

\begin{tabular}{|c|c|c|c|c|}
\hline Original & iTranslate & & Human & \\
\hline English & Spanish & Chinese & Spanish & Chinese \\
\hline $\begin{array}{l}\text { What are my blood sugar, } \\
\text { blood pressure, and choles- } \\
\text { terol numbers? }\end{array}$ & $\begin{array}{l}\text { ¿Cuáles son mis azúcar en } \\
\text { la sangre, presión arterial y } \\
\text { colesterol? }\end{array}$ & $\begin{array}{l}\text { 我的血糖、血压和胆固醇 } \\
\text { 是什么? }\end{array}$ & $\begin{array}{l}\text { ¿Cuáles son mis números de } \\
\text { azúcar en la sangre, presión } \\
\text { arterial y colesterol? }\end{array}$ & $\begin{array}{l}\text { 我的血糖、血压和胆固醇 } \\
\text { 的值是多少? }\end{array}$ \\
\hline What should they be? & ¿Qué deberían ser? & 他们应该是什么? & $\begin{array}{l}\text { ¿Cuáles deben de ser mis } \\
\text { números? }\end{array}$ & 正常值应该是多少? \\
\hline $\begin{array}{l}\text { What actions should I take } \\
\text { to reach these goals? }\end{array}$ & $\begin{array}{l}\text { ¿Qué medidas debo tomar } \\
\text { para alcanzar estas metas? }\end{array}$ & $\begin{array}{l}\text { 应该采取何种行动来达到 } \\
\text { 这些目标? }\end{array}$ & $\begin{array}{l}\text { ¿Qué debo de hacer para al- } \\
\text { canzar esas metas? }\end{array}$ & $\begin{array}{l}\text { 我应该怎么做来达到正常 } \\
\text { 值? }\end{array}$ \\
\hline
\end{tabular}

\section{Step 4. Evaluation}

We sent invitation emails to the first 12 English-Spanish translators and 12 English-Chinese ATA certified translators listed on the ATA website. We emailed the survey package to the first six translators (three Spanish and three Chinese respectively) who accepted our study invitation. We asked them to evaluate the two versions of the voice translations (one by iTranslate app and the other one by the professional translator). Each evaluator received a US \$15 check after submitting the evaluation survey package via email. The two interpreters who provided the human translation versions did not serve as evaluators, nor were they aware that their translations would be evaluated by other translators.

\section{Survey Package}

To minimize rater bias and blind the evaluation process, the audio files were marked as version 1 (sentences translated by iTranslate) and version 2 (sentences translated by a human).
The survey package contained one evaluation rubric in an MS Word file and two audio files (versions 1 and 2). We asked the evaluators to score each of the translated sentences using the evaluation rubric (see Table 2).

\section{Evaluation Rubric}

We adapted the evaluation rubric from Khana et al [22], instructed the raters to evaluate the translated sentences based on four criteria-Fluency, Adequacy, Meaning, and Severity - on a 5-point scale (1 indicates the lowest quality and 5 indicates the highest quality). The Fluency and Adequacy criteria are standard domains for assessing machine translation quality [25]. Fluency assesses readability, grammar, and understandability. Adequacy assesses the amount of original information preserved. Meaning assesses the equivalency of the translation and the original sentence and detects misleading information [26]. Severity assesses the degree of the negative impact on a patient's health outcome. Table 2 presents the four criteria and the description for each criterion.

Table 2. Rubric for evaluating translation quality.

\begin{tabular}{|c|c|c|c|c|}
\hline & Fluency & Adequacy & Meaning & Severity \\
\hline 1 & $\begin{array}{l}\text { No fluency; } \\
\text { no appreciable grammar, not under- } \\
\text { standable }\end{array}$ & $\begin{array}{l}0 \% \text { of information conveyed from } \\
\text { the original }\end{array}$ & Totally different meaning from the original & Dangerous to patient \\
\hline 2 & $\begin{array}{l}\text { Marginal fluency; } \\
\text { several grammatical errors }\end{array}$ & $\begin{array}{l}25 \% \text { of information conveyed from } \\
\text { the original }\end{array}$ & $\begin{array}{l}\text { Misleading information added/omitted com- } \\
\text { pared to the original }\end{array}$ & $\begin{array}{l}\text { Impairs care in some } \\
\text { way }\end{array}$ \\
\hline 3 & $\begin{array}{l}\text { Good fluency; } \\
\text { several grammatical errors, under- } \\
\text { standable }\end{array}$ & $\begin{array}{l}50 \% \text { of information conveyed from } \\
\text { the original }\end{array}$ & Partially the same meaning as the original & Delays necessary care \\
\hline 4 & $\begin{array}{l}\text { Excellent fluency; } \\
\text { few grammatical errors }\end{array}$ & $\begin{array}{l}75 \% \text { of information conveyed from } \\
\text { the original }\end{array}$ & Almost the same meaning as the original & $\begin{array}{l}\text { Unclear effect on pa- } \\
\text { tient care }\end{array}$ \\
\hline 5 & $\begin{array}{l}\text { Perfect fluency; } \\
\text { like reading a newspaper }\end{array}$ & $\begin{array}{l}100 \% \text { of information conveyed from } \\
\text { the original }\end{array}$ & Same meaning as the original & $\begin{array}{l}\text { No effect on patient } \\
\text { care }\end{array}$ \\
\hline
\end{tabular}

\section{Data Analysis}

We performed the Cronbach alpha test to assess the degree of rater agreement. Two sets of mean scores were calculated for each of the four domains (Fluency, Adequacy, Meaning, and Severity) on each sentence from the Chinese and Spanish rater groups. We also presented the readability statistics for each original English sentences. Readability statistics were generated using MS Word's Flesch-Kincaid Grade Level, which assesses the degree of difficulty for readers to understand a sentence or paragraph [27]. For ease of comparison, two sets of graphs shown in Figures 2 and 3 visually depict the translation quality between iTranslate app and the human interpreters starting from the easiest to the most difficult sentence based on the readability statistics. 
Figure 2. Scatterplots comparing Spanish iTranslate with the human translator scores.

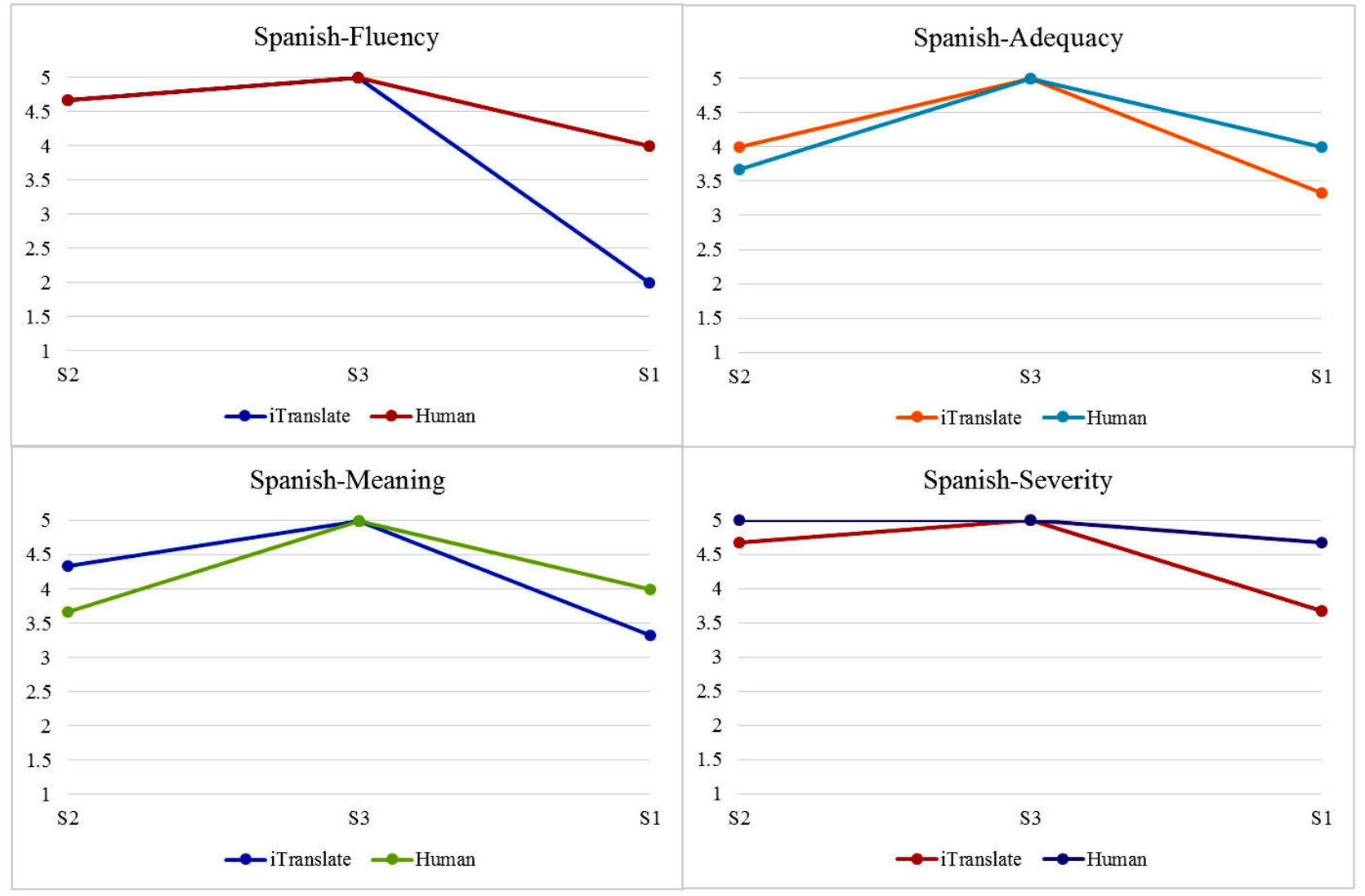

Figure 3. Scatterplots comparing Chinese iTranslate with the human translator scores.

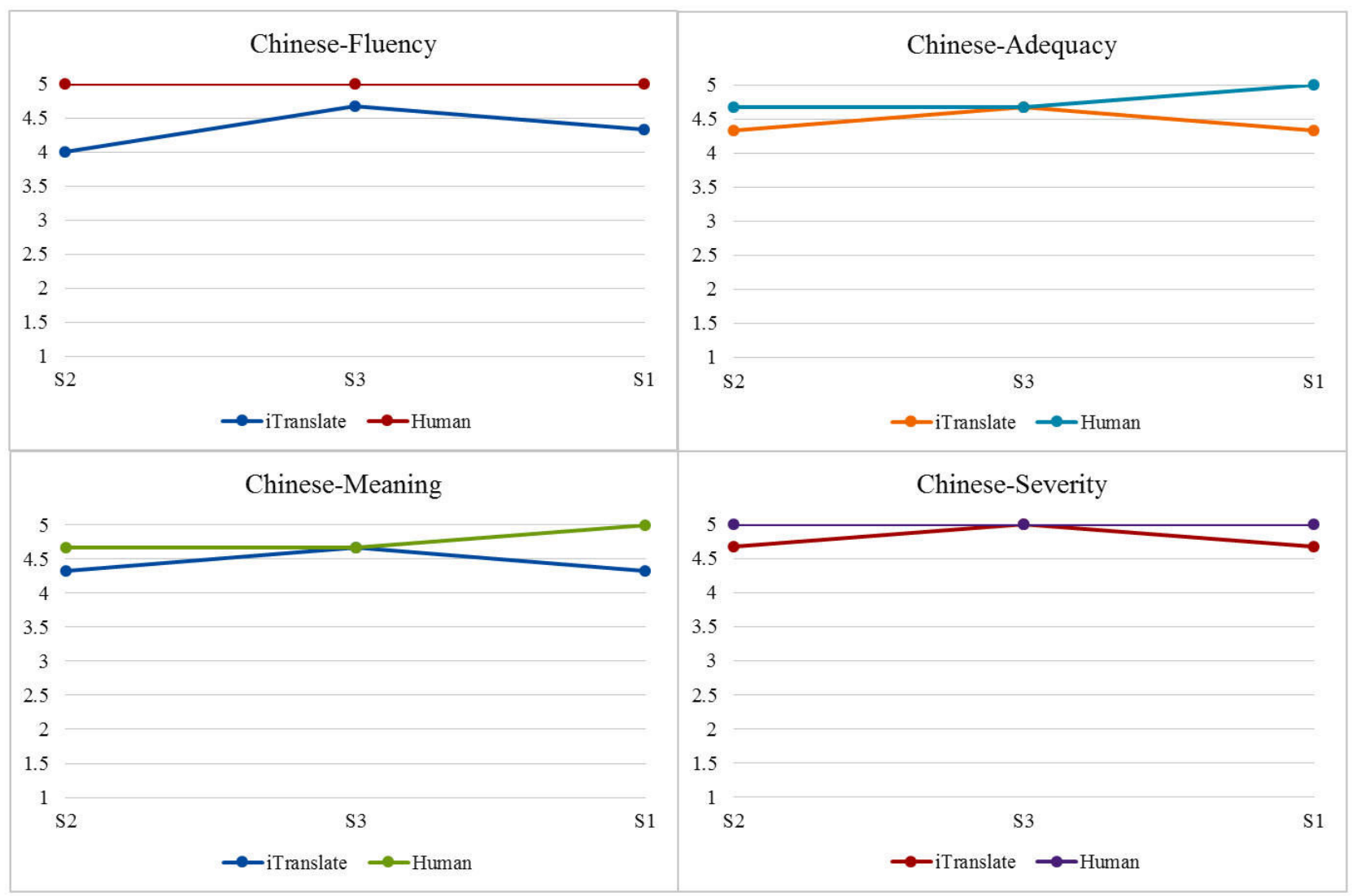




\section{Results}

\section{Interrater Reliability}

Cronbach alpha was used to assess the rating reliability across each evaluator. The Cronbach alpha values exhibited high degrees of agreement on the rating outcomes of both rater groups: .920 for the Spanish raters and .971 for the Chinese raters.

\section{Spanish Translation: iTranslate Versus Human}

We ranked the sentences based on their readability scores and presented the results with the easiest sentence first, followed by the medium, and put the most difficult sentence last (Table 3). Within the Fluency domain for iTranslate, the two relatively simple sentences (S2 and S3) had almost perfect fluency; however, the most difficult sentence (S1) had marginal fluency with several grammatical errors (Fluency=2). All the sentences translated by the Spanish human translator received excellent or perfect fluency scores (Fluency $\geq 4$ ). Within the Adequacy domain for iTranslate, S2 conveyed about $75 \%$ of the original information, S3 conveyed $100 \%$ of the original information, but $\mathrm{S} 1$ conveyed about half of the original information. All the sentences translated by the Spanish human translator conveyed most of the original information. Within the Meaning domain for iTranslate, S2 and S3 had almost the same meaning as the original, but $\mathrm{S} 1$ had practically the same meaning as the original. All the sentences translated by the Spanish human translator had almost the same meaning as the original. Within the Severity domain for iTranslate, S2 and S3 had almost no effect on patient care, but $\mathrm{S} 1$ had unclear effect on patient care. All the sentences translated by the Spanish human translator had (almost) no effect on patient care.

Table 3. Mean scores for Spanish iTranslate and the human Spanish translator.

\begin{tabular}{|c|c|c|c|c|c|c|c|c|c|}
\hline \multirow[t]{2}{*}{ Original sentences } & \multirow{2}{*}{$\begin{array}{l}\text { Flesch-Kincaid } \\
\text { grade level }\end{array}$} & \multicolumn{4}{|l|}{ iTranslate } & \multicolumn{4}{|l|}{ Human } \\
\hline & & Fluency & Adequacy & Meaning & Severity & Fluency & Adequacy & Meaning & Severity \\
\hline $\begin{array}{l}\text { S2. What should } \\
\text { they be? }\end{array}$ & 0.0 & 4.67 & 4 & 4.33 & 4.67 & 4.67 & 3.67 & 3.67 & 5 \\
\hline $\begin{array}{l}\text { S3. What actions } \\
\text { should I take to } \\
\text { reach these goals? }\end{array}$ & 1.0 & 5 & 5 & 5 & 5 & 5 & 5 & 5 & 5 \\
\hline $\begin{array}{l}\text { S1. What are my } \\
\text { blood sugar, blood } \\
\text { pressure, and } \\
\text { cholesterol num- } \\
\text { bers? }\end{array}$ & 7.1 & 2 & 3.33 & 3.33 & 3.67 & 4 & 4 & 4 & 4.67 \\
\hline
\end{tabular}

\section{Chinese Translation: iTranslate Versus Human}

As shown in Table 4, within the Fluency domain, all the sentences translated by both iTranslate and the Chinese human translator had excellent or perfect fluency. Within the Adequacy domain, all the sentences conveyed more than $75 \%$ to $100 \%$ of the original information. Within the Meaning domain, all the sentences had (almost) the same meaning as the original. Within the Severity domain, all the sentences had almost no effect on patient care.

Table 4. Mean scores for Chinese iTranslate and the human Chinese translator.

\begin{tabular}{|c|c|c|c|c|c|c|c|c|c|}
\hline \multirow[t]{2}{*}{ Original sentences } & \multirow{2}{*}{$\begin{array}{l}\text { Flesch-Kincaid } \\
\text { Grade Level }\end{array}$} & \multicolumn{4}{|l|}{ iTranslate } & \multicolumn{4}{|l|}{ Human } \\
\hline & & Fluency & Adequacy & Meaning & Severity & Fluency & Adequacy & Meaning & Severity \\
\hline $\begin{array}{l}\text { S2. What should } \\
\text { they be? }\end{array}$ & 0.0 & 4 & 4.33 & 4.33 & 4.67 & 5 & 4.67 & 4.67 & 5 \\
\hline $\begin{array}{l}\text { S3. What actions } \\
\text { should I take to } \\
\text { reach these goals? }\end{array}$ & 1.0 & 4.67 & 4.67 & 4.67 & 5 & 5 & 4.67 & 4.67 & 5 \\
\hline $\begin{array}{l}\text { S1. What are my } \\
\text { blood sugar, blood } \\
\text { pressure, and } \\
\text { cholesterol num- } \\
\text { bers? }\end{array}$ & 7.1 & 4.33 & 4.33 & 4.33 & 4.67 & 5 & 5 & 5 & 5 \\
\hline
\end{tabular}

\section{Visually Comparing iTranslate and Human Versions}

To better compare and capture the trends among sentences with regard to the quality scores on four domains, we created two graphs, presenting the findings of the easiest sentence (S2) first and the most difficult sentence (S1) last.
When sentences were translated from English to Spanish (Figure 2), for the easiest sentence (S2), there was a slight difference between iTranslate and the Spanish human translator, where iTranslate received slightly higher scores in the Adequacy (4 vs 3.67) and Meaning domains (4.33 vs 3.67), but slightly lower scores in the Severity domain (4.67 vs 5$)$. There was no 
difference between iTranslate app and the Spanish human translator on S3 (the medium difficult sentence) in any of the four domains (5). For the most difficult sentence (S1), there was a slight difference between iTranslate and the Spanish human in the Adequacy and Meaning domains, where iTranslate received slightly lower scores (3.33 vs 4). We also noticed some gaps for S1 in the Fluency and Severity domains, where iTranslate received lower scores ( 2 vs 4 and 3.67 vs 4.67 ).

As shown in Figure 3, when sentences were translated from English to Chinese, there was almost no difference between the ratings of iTranslate app and the Chinese human translator on S3 (the medium difficult sentence) in any of the four domains. This funding is similar to the Spanish language. For the easiest sentence (S2) and the most difficult sentence (S1), there was a slight difference between iTranslate and the Chinese human translator, where iTranslate received slightly lower scores in all the four domains.

\section{Discussion}

\section{Principal Findings}

This pilot study compared the translation quality of iTranslate and professional human translators using three questions drawn from a diabetes patient education pamphlet. Materials were translated from English to Spanish and Chinese (Mandarin). We found iTranslate generally provided translation quality comparable to human translators on simple and medium difficulty sentences. The voice recognition feature and voice outputs employed by iTranslate produced text quality, clarity, and auditory richness (voice quality: native accent, tone, inflection, and delivery), which benefits individuals who cannot read in their native languages. However, iTranslate tends to make more errors when translating difficult sentences.

When translating the easiest sentence (ie, S2 "What should they be?", Flesch-Kincaid Grade Level=0.0) from English to Spanish, the voice employed by iTranslate softened and deadened the [n] when pronouncing [deberían] so that the [n] almost sounds omitted. On the other hand, the Spanish human translator added the antecedent noun for the pronoun "they". Therefore, even though the professional translator received slightly lower scores on the Adequacy and Meaning compared to iTranslate, the Spanish human translator received a slightly higher score on the Severity compared to iTranslate. One of the Spanish raters believed that $\mathrm{S} 2$ translated by iTranslate from English to Spanish had an unclear effect on patient care. When translating it from English to Chinese, iTranslate made no errors. Compared to the literal translation by iTranslate, the Chinese human interpreter added some extra information to clarify the word "they", which translated the sentence into [正常值应该是多少?] (What should the normal range be?). Although iTranslate did not make any errors, the Chinese human version contained more specific and meaningful information. We believe this was the reason why iTranslate received slightly lower scores on all the four criteria compared to the Chinese human translator. Also, one of the Chinese raters believed that it had an unclear effect on patient care.
When translating the relatively easy sentence (ie, S3 "What actions should I take to reach these goals?", Flesch-Kincaid Grade Level=1.0) from English to Spanish, the Spanish raters agreed that both versions had no effect on patient care even though the Spanish human interpreter substituted those ["esas"] for these [estas"]. Both iTranslate and the Spanish human interpreter received full scores on every criterion. When translating it from English to Chinese, iTranslate omitted the word "I" and translated this sentence into ("What actions should be taken to reach these goals?"). In comparison, the Chinese human interpreter substituted the phrase "take actions" into "do" and specified "these goals" into "normal numbers." Therefore, the Chinese human interpreter translated S3 into [我 应该怎么做来达到正常值? ] ("What should I do to reach normal numbers?”). Neither iTranslate nor the human interpreter correctly translated S3 word for word; however, the general meaning of the original sentence has not been changed. Thus, all the raters agreed that $\mathrm{S} 3$ translated by either iTranslate or the Chinese human interpreter had no effect on patient care.

When translating the most difficult sentence (ie, S1 "What are my blood sugar, blood pressure, and cholesterol numbers?, Flesch-Kincaid Grade Level=7.1) from English to Spanish, iTranslate omitted the word "number." Therefore, the Spanish evaluators believed it had marginal fluency with several grammatical errors, conveyed about half of the original information, had practically the same meaning as the original, and had an unclear effect on patient care or delays necessary care. On the other hand, the Spanish human interpreter did not make any errors when translating S1. When translating it from English to Chinese, iTranslate made the exactly same error as translating it from English to Spanish-it omitted the word "number" as well. Therefore, this sentence did not received full scores on Fluency, Adequacy, and Meaning, which led to one of the Chinese evaluators' believing that such an error had an unclear effect on patient care. On the other hand, the Chinese human interpreter did not make any errors when translating S1. Interestingly, even though iTranslate made the exactly the same error on S1 for the Spanish and Chinese translations, the Spanish raters gave it lower scores on all the criteria than the Chinese raters did.

To minimize rater bias, we blinded the audio version of the translated question so that the raters could not identify the two audio versions (iTranslate and the human translations). However, rater bias might still exist because each rater had their interpretation of the evaluation rubric. Variability in the rating scores may result from bias (systematic error) or random error (unpredictable). For example, S1 translated by iTranslate from English to Spanish received lower scores on all the criteria than the same sentence translated by iTranslate from English to Chinese even though the Chinese and Spanish translations made the exact same error-omitting the head noun "numbers" in the nominal phrase "my blood sugar, blood pressure, and cholesterol numbers." Usually there are number of ways to correctly translate a sentence; however, individuals might have different preferences on evaluating translation quality. Another example is Fluency. According to the rubric, 4 represents "excellent fluency" and 5 represents "perfect fluency." We made no attempt to standardize the domain descriptors or train the raters; 
therefore, each evaluator might have a slightly different interpretation of "excellent" and "perfect." Therefore, although we can make broad statements about the comparability and adequacy based on the scoring rubric of the human and machine translation in each language, we cannot conclude that iTranslate produces more accurate translations from English to Chinese than from English to Spanish.

Our findings appear to support iTranslate as producing competent, understandable translations for simple sentences. However, once the sentences get more complicated, iTranslate tends to make more errors. Previous studies documented high rate of errors in machine translations when translating written sentences. For instance, Sharif and Tse [28] identified half of the medicine labels translated by computer programs from English to Spanish as being incorrect. In another study, Khanna et al [22] found that Google Translate made more errors compared to human translators when translating patient education texts from English to Spanish. Chen et al [21] evaluated the accuracy of Google Translate when translating diabetes patient education materials from English to multiple languages (Spanish and Chinese). The authors reported that Google produced more accurate translations from English to Spanish than English to Chinese [21]. Turner et al [23] also reported a high error rate when Google translated health websites from English to Chinese. One explanation for the difference between our findings and the evidence noted above might result from sentence difficulty. Interestingly, our findings indicated that iTranslate was a relatively comparable tool when translating simple spoken sentences from English to Spanish and Chinese. We propose that the machine translation quality was comparable to the human translations only when the sentence was easier to understand due to the simplicity of the grammatical constructions. Hence, our results support the findings of Zeng-Treitler et al [24] who found that machine translation tools appear to be less likely to provide accurate translation for longer and more difficult sentences.

Guidelines are available for health professionals to work with human interpreters in clinical encounters [29-32]; however, to date and to the best of our knowledge, there are no recommendations or guidelines about using mobile translation apps. Randhawa et al [33] pointed out that machine translation devices have several potential benefits in clinical settings such as helping clarify patient histories, reviewing a clinical diagnosis, restating the recommended treatment plan, and encouraging patients to ask questions. Based on previous machine translation commentary studies $[33,34]$ and our pilot data, we recommend that clinicians consider the following when interacting with LEP patients using mobile language translation apps as communication assistance tools: (1) use the mobile translation apps to supplement but not supplant human translators, and (2) provide information in clients' and caregivers' mother tongue about the mobile translation apps and how to use them, along with appropriate precautions.

\section{Limitations}

This pilot study has several limitations. First, this study assessed the quality of the iTranslate mobile language translation app using only three spoken sentences from a diabetes patient education pamphlet. To compensate for the small number of sentence units, we investigated translations of these sentences from English into two languages (Spanish and Chinese). Second, we assessed only Spanish and Chinese translations so that the findings should not be applied to other languages. Future studies should investigate multiple machine translation tools with a larger sentence sample drawn from other public health materials as well as conversations from real clinical encounters. It is necessary to further investigate the relationship between machine translation error patterns and sentence complexity levels. Also, more studies should explore the app using experience from patients with LEP in various languages.

\section{Conclusions}

To the best of our knowledge, this is the first study to evaluate and compare the quality of a mobile language translation app with a voice recognition feature and professional human translators. We found iTranslate could produce competent, understandable translations for simple sentences. However, once sentences became more complicated, iTranslate seemed to make more errors.

\section{Acknowledgments}

This study was supported by the College of Education and Human Development (CEHD) at Texas A\&M University under the CEHD Graduate Research Grant Award. We want to give sincere thanks to Dr. Bruce Thompson for his assistance in the data analysis process. We also thank all of the ATA translators who participated in this study.

\section{Conflicts of Interest}

None declared.

\section{Multimedia Appendix 1}

You are the heart of your family...take care of it.

\section{References}


1. Centers for Disease Control and Prevention. National Diabetes Statistics Report: Estimates of Diabetes and Its Burden in the United States, 2014. Atlanta, GA: US Department of Health and Human Services; 2014. URL: https://www.cdc.gov/ diabetes/pubs/statsreport14/national-diabetes-report-web.pdf [accessed 2017-06-22] [WebCite Cache ID 6rQ4cmUno]

2. Centers for Disease Control and Prevention. Centers for Disease Control and Prevention. 2015 Dec 01. Age-adjusted rates of diagnosed diabetes per 100 civilian, non-institutionalized population, by race and sex, United States, 1980-2014 URL: https://www.cdc.gov/diabetes/statistics/prev/national/figraceethsex.htm [accessed 2017-06-22] [WebCite Cache ID 6rQ58paXl]

3. Ryan C. US Census Bureau.: US Census Bureau; 2013 Aug. Language use in the United States: 2011 URL: https://www. census.gov/prod/2013pubs/acs-22.pdf [accessed 2017-06-21] [WebCite Cache ID 6rQ5dmo70]

4. Wilson E, Chen AHM, Grumbach K, Wang F, Fernandez A. Effects of limited English proficiency and physician language on health care comprehension. J Gen Intern Med 2005 Sep;20(9):800-806 [FREE Full text] [doi:

10.1111/j.1525-1497.2005.0174.x] [Medline: 16117746]

5. Toci E, Burazeri G, Jerliu N, Sørensen K, Ramadani N, Hysa B, et al. Health literacy, self-perceived health and self-reported chronic morbidity among older people in Kosovo. Health Promot Int 2015 Sep;30(3):667-674. [doi: 10.1093/heapro/dau009] [Medline: 24604915]

6. Ponce N, Hays R, Cunningham W. Linguistic disparities in health care access and health status among older adults. J Gen Intern Med 2006 Jul;21(7):786-791. [doi: 10.1111/j.1525-1497.2006.00491.x] [Medline: 16808783]

7. Nair E, Cienkowski K. The impact of health literacy on patient understanding of counseling and education materials. Int $\mathrm{J}$ Audiol 2010 Feb;49(2):71-75. [doi: 10.3109/14992020903280161] [Medline: 20151879]

8. Jacobs E, Karavolos K, Rathouz P, Ferris T, Powell L. Limited English proficiency and breast and cervical cancer screening in a multiethnic population. Am J Public Health 2005 Aug;95(8):1410-1416. [doi: 10.2105/AJPH.2004.041418] [Medline: 16043670]

9. Fernandez A, Schillinger D, Warton E, Adler N, Moffet HH, Schenker Y, et al. Language barriers, physician-patient language concordance, and glycemic control among insured Latinos with diabetes: the Diabetes Study of Northern California (DISTANCE). J Gen Intern Med 2011 Feb;26(2):170-176 [FREE Full text] [doi: 10.1007/s11606-010-1507-6] [Medline: 20878497]

10. Zheng Y, Lamoureux E, Chiang PCP, Anuar AR, Ding J, Wang JJ, et al. Language barrier and its relationship to diabetes and diabetic retinopathy. BMC Public Health 2012 Sep 13;12:781 [FREE Full text] [doi: 10.1186/1471-2458-12-781] [Medline: 22974298]

11. Poushter J. Pew Research Center. 2016 Feb 22. Smartphone ownership and Internet usage continues to climb in emerging economies URL: http://www.pewglobal.org/2016/02/22/ smartphone-ownership-and-internet-usage-continues-to-climb-in-emerging-economies/ [accessed 2017-06-22] [WebCite Cache ID 6rQ7JD6sD]

12. Anderson M. Pew Research Center. 2015 Aug 29. The demographics of device ownership URL: http://www.pewinternet.org/ 2015/10/29/the-demographics-of-device-ownership/ [accessed 2017-06-22] [WebCite Cache ID 6rQ7nzSQZ]

13. Anderson M. Pew Internet Research. 2015 Oct 29. Racial and ethnic differences in how people use mobile technology URL: http://www.pewresearch.org/fact-tank/2015/04/30/racial-and-ethnic-differences-in-how-people-use-mobile-technology/ [accessed 2017-06-22] [WebCite Cache ID 6rQ8eCIj]

14. Tahir D. Modern Healthcare. 2015. App breaks down language barriers for patients, doctors URL: http://www. modernhealthcare.com/article/20150124/MAGAZINE/301249980 [accessed 2017-06-22] [WebCite Cache ID 6rQ8opd0F]

15. Mirsa T. CITYLAB. 2014. Attention cities looking to engage immigrants: Don't dismiss the smartphone URL: $\underline{\text { https://www. }}$ citylab.com/solutions/2014/09/attention-cities-looking-to-engage-immigrants-dont-dismiss-the-smartphone/380229/ [accessed 2017-06-22] [WebCite Cache ID 6rQ8voAUL]

16. Butow P, Goldstein D, Bell M, Sze M, Aldridge LJ, Abdo S, et al. Interpretation in consultations with immigrant patients with cancer: how accurate is it? J Clin Oncol 2011 Jul 10;29(20):2801-2807. [doi: 10.1200/JCO.2010.34.3335] [Medline: 21670462]

17. Flores G, Laws M, Mayo S, Zuckerman B, Abreu M, Medina L, et al. Errors in medical interpretation and their potential clinical consequences in pediatric encounters. Pediatrics 2003 Jan;111(1):6-14. [Medline: 12509547]

18. Nápoles AM, Santoyo-Olsson J, Karliner L, Gregorich S, Pérez-Stable EJ. Inaccurate Language Interpretation and Its Clinical Significance in the Medical Encounters of Spanish-speaking Latinos. Med Care 2015 Nov;53(11):940-947 [FREE Full text] [doi: 10.1097/MLR.0000000000000422] [Medline: 26465121]

19. Abreu R, Adriatico T. Spanish for the Audiologist: Is There an App for That? SIG 14 Perspectives on Communication Disorders and Sciences in Culturally and Linguistically Diverse (CLD) Populations. Perspectives on Communication Disorders and Sciences in Culturally and Linguistically Diverse Populations 2015;22(3):122-128.

20. Albrecht U, Behrends M, Schmeer R, Matthies HK, von JU. Usage of multilingual mobile translation applications in clinical settings. JMIR Mhealth Uhealth 2013 Apr 23;1(1):e4 [FREE Full text] [doi: 10.2196/mhealth.2268] [Medline: 25100677]

21. Chen X, Acosta S, Barry A. Evaluating the Accuracy of Google Translate for Diabetes Education Material. JMIR Diabetes 2016 Jun 28;1(1):e3. [doi: 10.2196/diabetes.5848] 
22. Khanna RR, Karliner LS, Eck M, Vittinghoff E, Koenig CJ, Fang MC. Performance of an online translation tool when applied to patient educational material. J Hosp Med 2011 Nov;6(9):519-525. [doi: 10.1002/jhm.898] [Medline: 22034196]

23. Turner A, Dew K, Desai L, Martin N, Kirchhoff K. Machine Translation of Public Health Materials From English to Chinese: A Feasibility Study. JMIR Public Health Surveill 2015;1(2):e17 [FREE Full text] [doi: 10.2196/publichealth.4779] [Medline: 27227135]

24. Zeng-Treitler Q, Kim H, Rosemblat G, Keselman A. Can multilingual machine translation help make medical record content more comprehensible to patients? Stud Health Technol Inform 2010;160(Pt 1):73-77. [Medline: 20841653]

25. Eck M, Hori C. Overview of the IWSLT. 2005 Presented at: International Workshop on Spoken Language Translation; 2005; Pittsburg, PA.

26. Eck M, Hori C. Overview of the IWSLT. 2005 Presented at: International Workshop on Spoken Language Translation; 2005; Pittsburg, PA.

27. Stockmeyer N. Using Microsoft Word's readability program. Michigan Bar Journal 2009;88:46.

28. Sharif I, Tse J. Accuracy of computer-generated, spanish-language medicine labels. Pediatrics 2010 May;125(5):960-965 [FREE Full text] [doi: 10.1542/peds.2009-2530] [Medline: 20368321]

29. Hadziabdic E, Hjelm K. Working with interpreters: practical advice for use of an interpreter in healthcare. Int J Evid Based Healthc 2013 Mar;11(1):69-76. [doi: 10.1111/1744-1609.12005] [Medline: 23448332]

30. Juckett G, Unger K. Appropriate use of medical interpreters. Am Fam Physician 2014 Oct 01;90(7):476-480 [FRE Full text] [Medline: 25369625]

31. Kaur R, Oakley S, Venn P. Using face-to-face interpreters in healthcare. Nurs Times 2014;110(21):20-21. [Medline: 24960977]

32. Tribe R, Thompson K. Working with interpreters in health settings: Guidelines for psychologists. In: The British Psychological Society. Leicester, UK: The British Psychological Society; 2008.

33. Randhawa G, Ferreyra M, Ahmed R, Ezzat O, Pottie K. Using machine translation in clinical practice. Can Fam Physician 2013 Apr;59(4):382-383 [FREE Full text] [Medline: 23585608]

34. Gonzalez A. Jensen Localization. 5 tips to ensure the success of machine translation URL: http://www.jensen-localization.com/ blog/ [accessed 2017-06-21] [WebCite Cache ID 6rQDvsgMt]

\section{Abbreviations \\ ATA: American Translators Association \\ CDC: Centers for Disease Control and Prevention \\ LEP: limited English proficiency}

Edited by $G$ Eysenbach; submitted 03.02.17; peer-reviewed by A Turner, $Y$ Albalawi, $N$ Diviani, $H$ Akram; comments to author 15.04.17; revised version received 21.05.17; accepted 11.06.17; published 29.06.17

Please cite as:

Chen X, Acosta S, Barry AE

Machine or Human? Evaluating the Quality of a Language Translation Mobile App for Diabetes Education Material

JMIR Diabetes 2017;2(1):e13

URL: http://diabetes.jmir.org/2017/1/e13/

doi: $10.2196 /$ diabetes. 7446

PMID: 30291084

CXuewei Chen, Sandra Acosta, Adam E Barry. Originally published in JMIR Diabetes (http://diabetes.jmir.org), 29.06.2017. This is an open-access article distributed under the terms of the Creative Commons Attribution License (https://creativecommons.org/licenses/by/4.0/), which permits unrestricted use, distribution, and reproduction in any medium, provided the original work, first published in JMIR Diabetes, is properly cited. The complete bibliographic information, a link to the original publication on http://diabetes.jmir.org/, as well as this copyright and license information must be included. 